\title{
Post monsoon floristic inventory of Nagarparkar, District Tharparkar, Sindh, Pakistan
}

\author{
Mumtaz Ali Saand ${ }^{1,2}$, Ameer Ahmed Mirbahar ${ }^{1}$, Naeema Khatoon \\ Khaskheli ${ }^{2}$, Khalil Ahmed Ansari ${ }^{2}$, Shakeel Ahmed Khaskheli ${ }^{1}$, \\ Muhammad Mahmood-ur-Rehman Jamro ${ }^{3}$ and Muzafar Hussain \\ Sirohi ${ }^{2 *}$ \\ 1. Centre for Conservation and Biodiversity (CBC), Shah Abdul Latif University Khairpur-Pakistan \\ 2. Department of Botany, Shah Abdul Latif University Khairpur-Pakistan \\ 3. AZRI field station Khairpur, Pakistan Agricultural Research Council (PARC)-Pakistan \\ *Corresponding author's email: muzafar.sirohi@salu.edu.pk \\ Citation \\ Mumtaz Ali Saand, Ameer Ahmed Mirbahar, Naeema Khatoon Khaskheli, Khalil Ahmed Ansari, Shakeel \\ Ahmed Khaskheli, Muhammad Mahmood-ur-Rehman Jamro and Muzafar Hussain Sirohi. Post monsoon \\ floristic inventory of Nagarparkar, District Tharkarpar, Sindh, Pakistan. Pure and Applied Biology. Vol. 8, Issue \\ 1, pp968-976. http://dx.doi.org/10.19045/bspab.2019.80038
}

\begin{tabular}{|c|c|c|c|}
\hline Received: $12 / 12 / 2018$ & Revised: 07/03/2019 & Accepted: 11/03/2019 & Online First: $27 / 03 / 2019$ \\
\hline
\end{tabular}

\section{Abstract}

The monsoon rain brings a green carpet of landscape to Thar Desert including Karoonjhar mountains range. We explored Nagarparkar, District Tharparkar, Sindh to record post monsoon botanical inventory of the region. The study confirmed 89 plant species of 26 plant families. The vegetation was dominated by Poaceae (18 spp.) and Fabaceae (15 spp.) families. About two third of the families were represented by single species. The vegetation was rich with grasses and herbs making about $60 \%$ of the species while the rest includes tress shrubs and subshrubs. The lifespan analysis revealed the dominance of perennials. The life form spectrum shows the abundance of Phanerophytes (30\%), Chamaephyte (28\%) and Therophyte (25\%) across the region. The present study demonstrates vegetation variations triggered by monsoon down pours and indicates the presence of various microhabitats in the study area. The current inventory would be useful for further ecological studies and conservation of species and habitats. These records would help to detect invasive species, their distribution and other environmental impacts on plant species in future.

Keywords: Floristic inventory; Life form; Life-span, Nagraparkar; Plant habits; Thar Desert

\section{Introduction}

The flora is an assembled checklist of plant species which provides information about plant biodiversity of any specific geographic zone [1,2]. A floristic inventory not only reveals the identification and description of local and regional species but also offer the evidence of plant phenology, invasion of new species and vegetation stress [1]. The information may also be useful to understand the impact of climate change on regional vegetation and the distribution of species $[3,4]$. The valuable data records compiled through floras could be utilized for future reference [2]. The flora may range from a local to regional level casing varied habitats [1, 5-9]. Pakistan is rich in floristic diversity and consists around 5,700, both indigenous and alien, plant species of vascular plants [10]. Numerous studies demonstrated the inventories of floras and their importance in 
Pakistan [1, 10]. Additionally, several forest, desert and wetland floristic inventories have been conducted in different parts of Sindh Province [2, 11-13]. District Tharparkar, a part of the Thar Desert, is situated at the south-eastern part of Pakistan, at about $433 \mathrm{~km}$ form the capital city of Sindh, Karachi. Thar is not only a fertile desert with a unique flora and fauna but also attributed to stunting landscapes and cultural diversity, thus often referred as open air museum [14]. Nagarparkar is one of the Talukas of Tharparkar District stretched over 3,862 $\mathrm{km}^{2}$. It is located between Thar Desert and Rann of Kutch and contains the dramatic Karunjar Hills covered by enormous plant species [14]. However, the study area is tropical desert climate in nature, it is cooler than the rest of the Tharparkar. During monsoon it receives considerable quantity of rainfall in the month of July to September. April, May and June are the hottest months (max: temperatures $39^{\circ} \mathrm{C}$ and min: $20^{\circ} \mathrm{C}$ ) and January and February are the coldest (max: temperatures $26^{\circ} \mathrm{C}$ and min: $6^{\circ} \mathrm{C}$ ). After the rains in August it becomes extremely green and pleasant and temperatures become bearable (max: temperature $34^{\circ} \mathrm{C}$ and min: $13^{\circ} \mathrm{C}$ ) [15]. This study was conducted to record the post monsoon floristic inventory of the area to observe plant biodiversity of Nagarparkar which is the part of Thar Desert, Sindh, Pakistan. The study provides information of flora of study area after it receives the monsoon rain and demonstrated the checklist of flora, their life-spans, life forms and habits.

\section{Materials and methods}

The study area was visited in post monsoon season 2017 and 2018. The plant species were collected from varied habitats e.g. sand dunes and rocky area of Nagarparkar. All collected specimens were processed for making herbarium and determination of their life-span and life form categories as suggested [2]. The identification of species was carried out with keys published in literature, i.e. Flora of Pakistan [16-18],
Flora of Karachi [19] and floral studies at Nara Desert [20]. The species were deposited in the Herbarium of Centre for Biodiversity and Conservation (CBC), Shah Abdul Latif University (SALU), Khairpur, Sindh for record. To get vernacular names of taxa, the local people of study area were interviewed. Raunkiaer's system [21] of classification was used to determine the life forms of the species. This system uses the position of perennating buds to determine the life form of a species.

\section{Results and discussion}

A total of 89 plant species belonging to 68 genera and 26 families were identified from study area (Table 1) Plant family Poaceae (14 gen., 18 spp.) was found predominant with high species richness, followed by the families Fabaceae (9 gen., 15 spp.), Malvaceae (3 gen., 6 spp.) and Asteraceae (3 gen., 5 spp.) (Figure 1). About 50\% of the identified families contained two to four species each, while another $35 \%$ were represented by single species (Table 1 ). Of 26 identified families, Poaceae and Cyperaceae, making less than $20 \%$ of species, represented Monocot clad while a high proportion of the species characterized Dicot clad (Figure 2a).

Previous studies had unveiled the floristic inventories including deserts and mountain regions of Sindh province [11-13, 20-24]. The first ever report of floristic inventory of Thar Desert was conducted by Chauudri [22] that revealed 122 plant species. This study confirmed $72 \%$ of those species in a part of that desert. This can be attributes to the varied microhabitats in the Nagarparkar District. Moreover, Thar Desert had been found to be dominated by plant families Amaranthaceae, Cucrbitaceae and Euphorbiaceae [25]. In contrast, this study confirmed the higher species richness of families Poaceae and Fabaceae (Figure 1). One reason for this contrast may be the recent changes in positions of genera in plant families. This study follows the recent classification suggested in the online Flora of Pakistan [26]. 
This study recorded Acacia senegal (Fabaceae); interestingly the same plant species is absent in Nara Desert, Khairpur [20]. Moreover, Aerva javanica (Fabaceae family), Leptadenia pyrotechnica. (Apocynaceae family), Calotropis procera (Apocynaceae family) and Prosopis cineraria (Fabaceae family) were found the most frequent species at sand dunes. Whereas the Prosopis glandulosa and Prosopis multiflora (Fabaceae family) were found abundant at rocky areas of Karoonjhar mountain. These species provide ecosystem services and play a role in human wellbeing $[23,27]$.

Moreover, the flora was rich with a high proportion of herbs and grasses making about $60 \%$ of the total species (Figure 3 ). This may explain the upshot of monsoon resulting in a growth of annual herbs. This is in consensus with Yaseen et al. [25] as they reported the $74 \%$ herbs, $18 \%$ shrubs and $8 \%$ trees, in ethnobotanical survey of Thar, desert of Sindh. The flora of the study area had only two, annual (37\%) and perennial (63\%), life spans (Figure 2b). However, Convolvulus glomeratus (Convolvulaceae) and Corchorus olitorius (Malvaceae) are contained within perennial and annual life span in this study, respectively, both species sometimes show biennial behaviour. The plant species belonging to four families, Cyperaceae, Limeaceae, Neuradaceae and Zygophyllaceae, had only annual life-span. On contrast, nine families (of 26) revealed perennial nature while the rest $(46 \%)$ of families had both, annual and perennial, natured species. The dominancy of herbs is evident after monsoon downpours (Figure 3). Similar results have been reported from other parts of Pakistan $[2,12]$.

According to Raunkiaer's life form spectrum [21], the vegetation was dominated by Phanerophytes (30\%), Chamaephyte (28\%) and Therophyte (25\%), while Hemicryptophyte and Xeropsammophyte were poorly represented (Figure 4). Though the percentage of various life form in neighboring desert areas varied, a high representation of Therophytes and Phanerophytes have been recorded in the Jaisalmer, Ajmer, Sariska and other parts of Rajasthan, India [28]. A high representation of Phanerophytes in the study may indicate the humid bioclimate of the area [29]. On the other hand, Therophytes, having a short life cycle, are mostly annuals and often grow in rainy season especially monsoon [29].

The monsoon rain provides enough water for the annual herbs and grasses to flourish in the study area (Figure 2). The herbs abundantly grow around Karoonjhar hills of the Nagarparkar [14]. This type of vegetation may raise on the water temporarily adsorbed in the top soil layer, synchronic to precipitation. Further, this is supported with the added layer of moisture present in the subsurface soil and the sandstone laying beneath the soil. However, the dominance of perennial may indicate the resistance of species towards the harsh climate or access to plentiful moisture for long periods after monsoon rains.

Table 1. Information of plant species distributed in Nagarparkar, Sindh. The position of various genera has been updated after the publication of flora of Pakistan. The legitimate name and plant families are updated with the new online version of flora of Pakistan [26]

\begin{tabular}{|c|c|c|c|c|c|c|}
\hline No. & Plant Species & $\begin{array}{c}\text { Local } \\
\text { name }\end{array}$ & Family & Habit & Life-span & Life form \\
\hline 1 & Abutilon indicum (L.) Sweet & Pat-teer & Malvaceae & Shrub & Perennial & Phanerophyte \\
\hline 2 & Acacia jacquemontii Benth. & Banwar & Fabaceae & Shrub & Perennial & Phanerophyte \\
\hline 3 & Acacia nilotica (L.) Delile & $\begin{array}{c}\text { Sindhi ba- } \\
\text { bur }\end{array}$ & Fabaceae & Tree & Perennial & Phanerophyte \\
\hline 4 & $\begin{array}{c}\text { Acacia senegal (L.) willd (Benth.) } \\
\text { Brenan }\end{array}$ & Kumbat & Fabaceae & Tree & Perennial & Phanerophyte \\
\hline
\end{tabular}




\begin{tabular}{|c|c|c|c|c|c|c|}
\hline 5 & $\begin{array}{l}\text { Aerva javanica var. javanica } \\
\text { (Burm.f.) Juss. ex. }\end{array}$ & Booh & $\begin{array}{l}\text { Amaran- } \\
\text { thaceae }\end{array}$ & Herb & Perennial & $\begin{array}{l}\text { Xeropsammo- } \\
\text { phyte }\end{array}$ \\
\hline 6 & Albizia lebbeck (L) Beth. & Sarenh & Fabaceae & Tree & Perennial & Phanerophyte \\
\hline 7 & Alhagi maurorum Medic. & Kandero & Fabaceae & Herb & Perennial & Chamaephyte \\
\hline 8 & Amaranthus graecizans L. & Marero & $\begin{array}{l}\text { Amaran- } \\
\text { thaceae }\end{array}$ & Herb & Annual & Therophyte \\
\hline 9 & Amaranthus viridis $\mathrm{L}$. & Lulur & $\begin{array}{l}\text { Amaran- } \\
\text { thaceae }\end{array}$ & Herb & Annual & Therophyte \\
\hline 10 & Aristida adscensionis $\mathrm{L}$. & $\begin{array}{l}\text { Lumb } \\
\text { Gaah }\end{array}$ & Poaceae & Grass & Annual & Therophyte \\
\hline 11 & Azadirachta indica A. Juss. & $\mathrm{Nim}$ & Meliaceae & Tree & Perennial & Phanerophyte \\
\hline 12 & Barleria prionitis L. & Khussaro & Acanthaceae & Shrub & Perennial & Chamaephyte \\
\hline 13 & $\begin{array}{l}\text { Boerhavia procumbens Banks ex } \\
\text { Rxb. }\end{array}$ & Dakhri & $\begin{array}{l}\text { Nyctagina- } \\
\text { ceae }\end{array}$ & Herb & Perennial & Chamaephyte \\
\hline 14 & Brachiaria ramosa $($ L.) Stapf & Sawri & Poaceae & Grass & Annual & Therophyte \\
\hline 15 & $\begin{array}{c}\text { Brachiaria reptans (L.) Gardner \& } \\
\text { Hubbard } \\
\end{array}$ & Sawri & Poaceae & Grass & Annual & Therophyte \\
\hline 16 & $\begin{array}{c}\text { Calotropis procera (Aiton) W. T, } \\
\text { Aiton }\end{array}$ & $\mathrm{Ak}$ & $\begin{array}{l}\text { Apocyna- } \\
\text { ceae }\end{array}$ & Shrub & Perennial & Phanerophyte \\
\hline 17 & $\begin{array}{c}\text { Capparis decidua (Forssk.) } \\
\text { Edgew. }\end{array}$ & Kirar & $\begin{array}{l}\text { Capparida- } \\
\text { ceae }\end{array}$ & Shrub & Perennial & Phanerophyte \\
\hline 18 & Cassia italica (Mill.) Spreng. & Ghora wal & Fabaceae & Herb & Annual & Therophyte \\
\hline 19 & Cenchrus biflorus Roxb. & Bhorut & Poaceae & Grass & Annual & Therophyte \\
\hline 20 & $\begin{array}{l}\text { Citrullus colocynthis (Linn.) } \\
\text { Schrad. }\end{array}$ & Trooh & $\begin{array}{c}\text { Cucurbita- } \\
\text { ceae }\end{array}$ & Herb & Perennial & $\begin{array}{c}\text { Hemicrypto- } \\
\text { phyte }\end{array}$ \\
\hline 21 & Cleome scaposa DC. & Kano gah & Cleomaceae & Herb & Annual & Therophyte \\
\hline 22 & Coccinia grandis (L.) Voigt. & Kanduri & $\begin{array}{l}\text { Cucurbita- } \\
\text { ceae }\end{array}$ & Herb & Perennial & Chamaephyte \\
\hline 23 & Commicarpus boissieri Cufod. & Ruper & $\begin{array}{l}\text { Nyctagina- } \\
\text { ceae }\end{array}$ & Herb & Perennial & $\begin{array}{c}\text { Xeropsammo- } \\
\text { phyte }\end{array}$ \\
\hline 24 & $\begin{array}{l}\text { Commiphora stocksiana (Engl.) } \\
\text { Engl. }\end{array}$ & Gugur & Burseraceae & $\begin{array}{l}\text { Large } \\
\text { Shrub }\end{array}$ & Perennial & Phanerophyte \\
\hline 25 & $\begin{array}{c}\text { Commiphora wightii (Arn.) } \\
\text { Bhandari }\end{array}$ & Gugur & Burseraceae & $\begin{array}{l}\text { Large } \\
\text { Shrub }\end{array}$ & Perennial & Phanerophyte \\
\hline 26 & Convolvulus arvensis $\mathrm{L}$ & Narro & $\begin{array}{c}\text { Convolvu- } \\
\text { laceae }\end{array}$ & Herb & Annual & Chamaephyte \\
\hline 27 & Convolvulus glomeratus Choisy & Sankavli & $\begin{array}{c}\text { Convolvu- } \\
\text { laceae }\end{array}$ & Herb & Perennial & Chamaephyte \\
\hline 28 & $\begin{array}{c}\text { Convolvulus rhyniospermus } \\
\text { Choisy }\end{array}$ & $\begin{array}{l}\text { Phesura } \\
\text { wal }\end{array}$ & $\begin{array}{c}\text { Convolvu- } \\
\text { laceae }\end{array}$ & Herb & Annual & Therophyte \\
\hline 29 & Corchorus depressus Stocks. & Mundheri & Malvaceae & Herb & Perennial & Chamaephyte \\
\hline 30 & Corchorus olitorius L. & Datehri & Malvaceae & Herb & Annual & Therophyte \\
\hline 31 & $\begin{array}{c}\text { Cordia gharaf (Forssk.) Ehrenb. } \\
\text { Ex Asch. }\end{array}$ & Leyar & $\begin{array}{l}\text { Boragina- } \\
\text { ceae }\end{array}$ & $\begin{array}{c}\text { Small } \\
\text { tree }\end{array}$ & Perennial & Phanerophyte \\
\hline 32 & $\begin{array}{c}\text { Crotalaria burhia } \text { Buch.-Ham. ex } \\
\text { Benth. }\end{array}$ & Chag & Fabaceae & $\begin{array}{l}\text { sub- } \\
\text { Shsrub }\end{array}$ & $\begin{array}{l}\text { Semi per- } \\
\text { ennial }\end{array}$ & Chamaephyte \\
\hline 33 & $\begin{array}{c}\text { Cymbopogon commutatus (Steud.) } \\
\text { stapf }\end{array}$ & Katan & Poaceae & Grass & Perennial & $\begin{array}{c}\text { Hemicrypto- } \\
\text { phyte }\end{array}$ \\
\hline 34 & $\begin{array}{c}\text { Cymbopogon jwarancusa (Jones) } \\
\text { Schult. }\end{array}$ & Katan & Poaceae & Grass & Perennial & $\begin{array}{l}\text { Xeropsammo- } \\
\text { phyte }\end{array}$ \\
\hline 35 & Cynodon dactylon (L.) Pers. & Chhabar & Poaceae & Grass & Perennial & Chamaephyte \\
\hline 36 & Cyperus rotundus $\mathrm{L}$. & Kabah & Cyperaceae & Sedge & Annual & Chamaephyte \\
\hline 37 & $\begin{array}{c}\text { Dactyloctenium aegyptium (L.) } \\
\text { Wild }\end{array}$ & $\begin{array}{l}\text { Gandheer } \\
\text { Gaah }\end{array}$ & Poaceae & Grass & Annual & Chamaephyte \\
\hline 38 & $\begin{array}{l}\text { Desmostachya bipinnata (L.) } \\
\text { Stapf. }\end{array}$ & Drabh & Poaceae & Grass & Perennial & Chamaephyte \\
\hline 39 & $\begin{array}{c}\text { Dichanthium annulatum (Forssk.) } \\
\text { Stapf }\end{array}$ & Palwan & Poaceae & Grass & Perennial & Chamaephyte \\
\hline
\end{tabular}




\begin{tabular}{|c|c|c|c|c|c|c|}
\hline 40 & Digera muricata (L.) Mart. & Lulur & $\begin{array}{l}\text { Amaran- } \\
\text { thaceae }\end{array}$ & Herb & Annual & Therophyte \\
\hline 41 & Digitaria ciliaris (Retz.) Koel. & $\begin{array}{l}\text { Pachar } \\
\text { Gaah }\end{array}$ & Poaceae & Grass & Annual & Therophyte \\
\hline 42 & Dipterygium glaucum Decne. & Phair & Cleomaceae & $\begin{array}{l}\text { sub- } \\
\text { shrub }\end{array}$ & Perennial & Phanerophyte \\
\hline 43 & Eclipta prostrata (L.) L. & $\begin{array}{c}\text { Daryai } \\
\text { buti }\end{array}$ & Asteraceae & Herb & Annual & $\begin{array}{l}\text { Hemicrypto- } \\
\text { phyte }\end{array}$ \\
\hline 44 & Euphorbia caducifolia Haines & Thohar & $\begin{array}{l}\text { Euphorbia- } \\
\text { ceae }\end{array}$ & Shrub & Perennial & Chamaephyte \\
\hline 45 & Euphorbia hirta L. & $\begin{array}{l}\text { Kheer } \\
\text { Wal }\end{array}$ & $\begin{array}{l}\text { Euphorbia- } \\
\text { ceae }\end{array}$ & Herb & Annual & Therophyte \\
\hline 46 & Euphorbia prostrata Aiton & $\begin{array}{l}\text { Kheera } \\
\text { Wal }\end{array}$ & $\begin{array}{l}\text { Euphorbia- } \\
\text { ceae }\end{array}$ & Herb & Annual & Therophyte \\
\hline 47 & $\begin{array}{l}\text { Fagonia indica var. schweinfurthii } \\
\text { Hadidi }\end{array}$ & Dramaaho & $\begin{array}{l}\text { Zygophyl- } \\
\text { laceae }\end{array}$ & $\begin{array}{l}\text { Sub- } \\
\text { shrub }\end{array}$ & Annual & $\begin{array}{l}\text { Xeropsammo- } \\
\text { phyte }\end{array}$ \\
\hline 48 & Grangea maderaspatana (L.) Poir. & Mundi & Asteraceae & Herb & Annual & Therophyte \\
\hline 49 & Grewia erythraea Schweinf. & Gangi & Malvaceae & Shrub & Perennial & Phanerophyte \\
\hline 50 & Grewia tenax (Forssk.) fiori. & Gangi & Malvaceae & Shrub & Perennial & Phanerophyte \\
\hline 51 & Grewia villosa Willd. & Gangi & Malvaceae & Shrub & Perennial & Phanerophyte \\
\hline 52 & Heliotropium crispum Desf. & Kharsan & $\begin{array}{l}\text { Boragina- } \\
\text { ceae }\end{array}$ & Herb & Annual & $\begin{array}{l}\text { Xeropsammo- } \\
\text { phyte }\end{array}$ \\
\hline 53 & Indigofera argentea Burm.f. & Lathio & Fabaceae & $\begin{array}{l}\text { Under- } \\
\text { shrub }\end{array}$ & Perennial & Therophyte \\
\hline 54 & $\begin{array}{l}\text { Indigofera cordifolia Heyne ex } \\
\text { Roth }\end{array}$ & Lathio & Fabaceae & Herb & Annual & Therophyte \\
\hline 55 & $\begin{array}{l}\text { Launaea procumbens (Roxb) Ra- } \\
\text { mayya \& Rajagopal. }\end{array}$ & Bhattar & Asteraceae & Herb & Perennial & $\begin{array}{l}\text { Hemicrypto- } \\
\text { phyte }\end{array}$ \\
\hline 56 & $\begin{array}{c}\text { Leptadenia pyrotechnica (Forsk.) } \\
\text { Decne. }\end{array}$ & Khip & $\begin{array}{l}\text { Apocyna- } \\
\text { ceae }\end{array}$ & Shrub & Perennial & Phanerophyte \\
\hline 57 & $\begin{array}{c}\text { Limeum indicum Stocks.ex T. An- } \\
\text { derson }\end{array}$ & Khir wal & Limeaceae & Herb & Annual & Therophyte \\
\hline 58 & Merremia aegyptia (L.) Urb. & Naaro & $\begin{array}{l}\text { Convolvu- } \\
\text { laceae }\end{array}$ & Herb & Perennial & Therophyte \\
\hline 59 & Moringa oleifera Lam. & Moa & Moringaceae & $\begin{array}{c}\text { Small } \\
\text { tree }\end{array}$ & Perennial & Phanerophyte \\
\hline 60 & $\begin{array}{l}\text { Mukia maderaspatana (L.) M. } \\
\text { Roem. }\end{array}$ & & $\begin{array}{l}\text { Cucurbita- } \\
\text { ceae }\end{array}$ & Herb & Annual & $\begin{array}{l}\text { Hemicrypto- } \\
\text { phyte }\end{array}$ \\
\hline 61 & Neurada procumbens L. & $\begin{array}{c}\text { Chha- } \\
\text { pri/Chhipr } \\
\text { i }\end{array}$ & Neuradaceae & Herb & Annual & Therophyte \\
\hline 62 & $\begin{array}{l}\text { Ochthochloa compressa (Forssk.) } \\
\text { Hilu. }\end{array}$ & Ghander & Poaceae & Grass & Perennial & $\begin{array}{l}\text { Hemicrypto- } \\
\text { phyte }\end{array}$ \\
\hline 63 & Oxystelma esculentum (L.f.) R. Br. & Phuli & $\begin{array}{l}\text { Apocyna- } \\
\text { ceae }\end{array}$ & Herb & Perennial & Chamaephyte \\
\hline 64 & Pennisetum orientale Rich. & Bhurut & Poaceae & Grass & Perennial & $\begin{array}{l}\text { Hemicrypto- } \\
\text { phyte }\end{array}$ \\
\hline 65 & $\begin{array}{c}\text { Phragmites karka (Retz.) Trin. ex } \\
\text { Steud. }\end{array}$ & Nar/Naro & Poaceae & Grass & Perennial & Chamaephyte \\
\hline 66 & $\begin{array}{l}\text { Pluchea lanceolata (DC.) C. B. } \\
\text { Clarke }\end{array}$ & Phaar Buti & Asteraceae & $\begin{array}{l}\text { Under- } \\
\text { shrub }\end{array}$ & Perennial & Chamaephyte \\
\hline 67 & Prosopis cineraria (L) Druce & Kandi & Fabaceae & Tree & Perennial & Phanerophyte \\
\hline 68 & Prosopis glandulosa Torr. & Devi & Fabaceae & Tree & Perennial & Phanerophyte \\
\hline 69 & Prosopis juliflora (Sw.) DC. & Devi & Fabaceae & Shrub & Perennial & Phanerophyte \\
\hline 70 & Rhynchosia minima (L.) DC. & Wanverhi & Fabaceae & Herb & Perennial & Chamaephyte \\
\hline 71 & Ricinus communis L. & Heran & $\begin{array}{l}\text { Euphorbia- } \\
\text { ceae }\end{array}$ & Shrub & Perennial & Phanerophyte \\
\hline 72 & Saccharum bengalense Retz. & Booro & Poaceae & Grass & Perennial & Chamaephyte \\
\hline 73 & Saccharum griffithii Munro ex & Kanh & Poaceae & Grass & Perennial & Phanerophyte \\
\hline
\end{tabular}


Saand et al.

\begin{tabular}{|c|c|c|c|c|c|c|}
\hline & Boiss. & & & & & \\
\hline 74 & Saccharum spontaneum L. & $\begin{array}{l}\text { Booro/Mu } \\
\text { nian }\end{array}$ & Poaceae & Grass & Perennial & Chamaephyte \\
\hline 75 & Salvadora oleoides Decne. & Jar/Peroon & $\begin{array}{l}\text { Solvador- } \\
\text { aceae }\end{array}$ & Shrub & Perennial & Phanerophyte \\
\hline 76 & Sesuvium sesuvioides Verdc. & Kori lonak & Aizoaceae & Herb & Annual & Therophyte \\
\hline 77 & Solanum surattense Burm.f. & $\begin{array}{l}\text { Kanderi } \\
\text { Wal }\end{array}$ & Solanaceae & hrub & Annual & Chamaephyte \\
\hline 78 & $\begin{array}{c}\text { Stipagrostis plumosa (L.) Munro } \\
\text { ex T. Anderson. }\end{array}$ & $\begin{array}{l}\text { Lumb } \\
\text { Gaah }\end{array}$ & Poaceae & Grass & Annual & Therophyte \\
\hline 79 & Tamarix aphylla (L.) Karst. & Lawo & $\begin{array}{l}\text { Tamarica- } \\
\text { ceae }\end{array}$ & Tree & Perennial & Phanerophyte \\
\hline 80 & Tamarix dioica Roxb. Ex Roth. & Lawo & $\begin{array}{l}\text { Tamarica- } \\
\text { ceae }\end{array}$ & Shrub & Perennial & Phanerophyte \\
\hline 81 & Tamarix indica willd. & Lai & $\begin{array}{l}\text { Tamarica- } \\
\text { ceae }\end{array}$ & Shrub & Perennial & Phanerophyte \\
\hline 82 & Tephrosia falciformis Ramasw. & ----------- & Fabaceae & Shrub & Perennial & Phanerophyte \\
\hline 83 & Tephrosia uniflora Pers. & Andhari & Fabaceae & Herb & Annual & Chamaephyte \\
\hline 84 & Trianthema portulacastrum L. & Waho & Aizoaceae & Herb & Annual & Therophyte \\
\hline 85 & Tribulus longipetalus Viv. & $\begin{array}{l}\text { Bakhro/B } \\
\text { hurt }\end{array}$ & $\begin{array}{l}\text { Zygophyl- } \\
\text { laceae }\end{array}$ & Herb & Annual & $\begin{array}{l}\text { Hemicrypto- } \\
\text { phyte }\end{array}$ \\
\hline 86 & Vernonia cinerascens Sch. Bip & Lanski & Asteraceae & Shrub & Perennial & Chamaephyte \\
\hline 87 & Withania somnifera (L.) Dunal & Akri & Solanaceae & Shrub & Perennial & Chamaephyte \\
\hline 88 & Zaleya pentandra (L.) Jeffrey. & Waho & Aizoaceae & Herb & Perennial & Chamaephyte \\
\hline 89 & $\begin{array}{l}\text { Ziziphus nummularia (Burm.f.) } \\
\text { Wt. \& A. }\end{array}$ & Ber & Phamnaceae & Shrub & Perennial & $\begin{array}{l}\text { Xeropsammo- } \\
\text { phyte }\end{array}$ \\
\hline
\end{tabular}

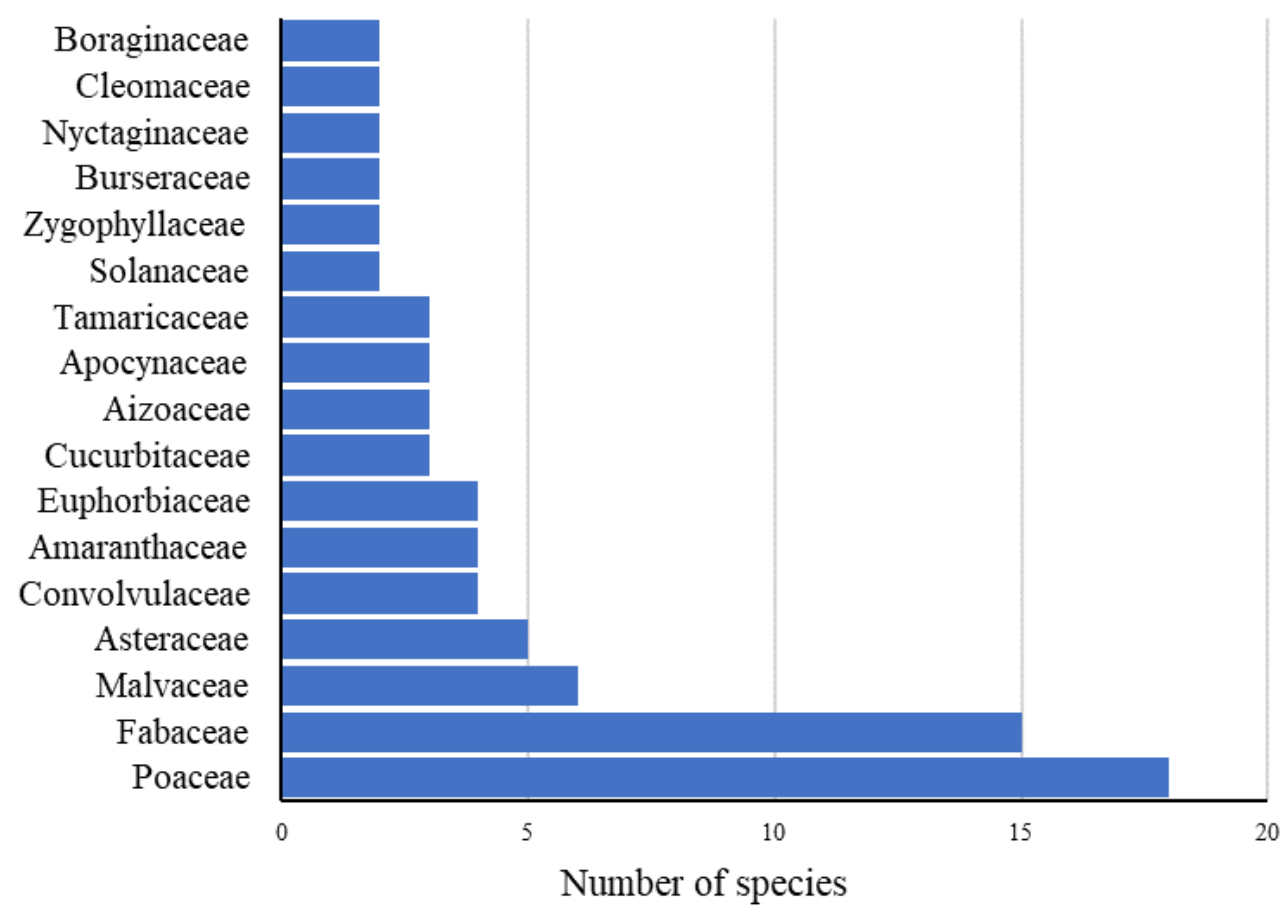

Figure 1. Plant families and species richness of flora of Nagarparker Sindh. Plant families Meliaceae, Cyperaceae, Acanthaceae, Limeaceae, Cappridaceae, Moringaceae, Phamnaceae, Neuradaceae, Solvadoraceae were represented by single species each therefore excluded from the figure. 


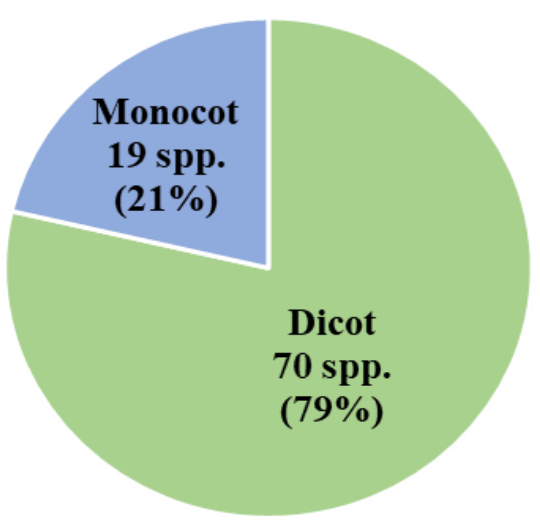

(a) Clad of species

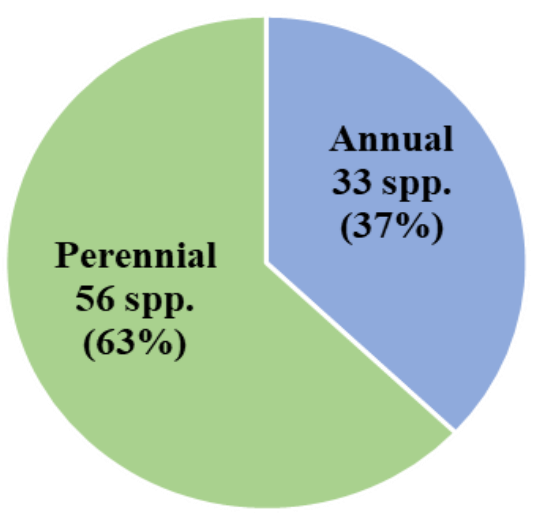

(b) Life span of species

Figure 2. Vegetation characteristics of flora of Nagarparker, Sindh (a) Clad of the species and (b) Life span of the species

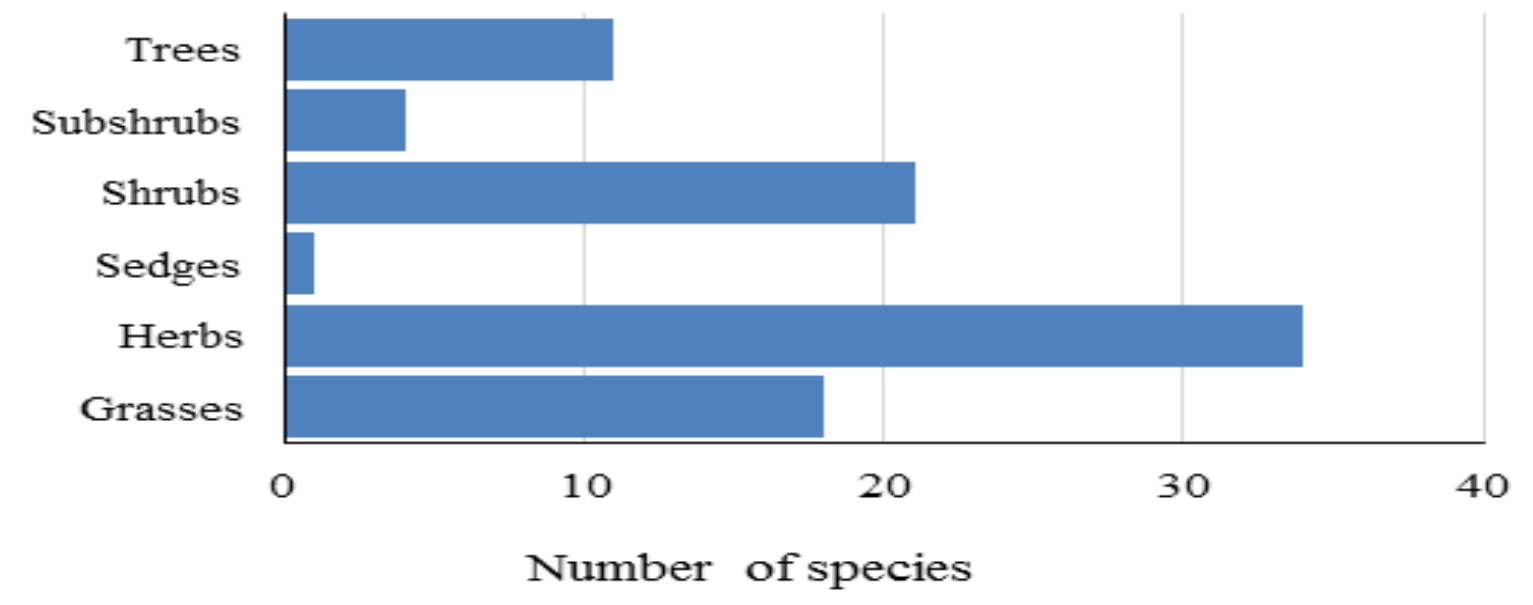

Figure 3. Plant habits of the species of the flora of Nagarparker, Sindh

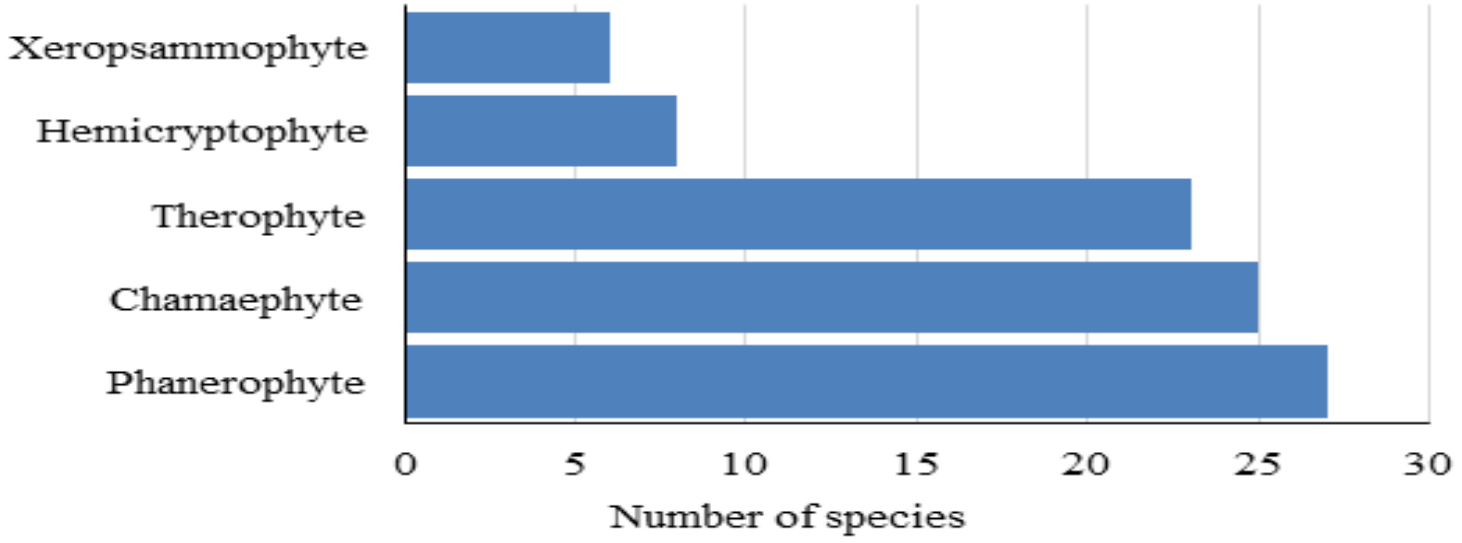

Figure 4. Life form of species identified in the flora of Nagarparker, Sindh 


\section{Conclusion}

The monsoon season embellishes the $\mathrm{Na}$ garparkar region, District Tharparkar, through addition of plants in its existing vegetation cover. The study recorded an inventory of 89 species of 26 plant families in post monsoon season. The vegetation cover was dominated by the species of family Poaceae and Malvaceae. The diversity in the life forms of the species was supported by a wide range of microhabitats present in the study area. The study will serve as a comprehensive inventory of flowering plant to perceive the potential threats of invasive species and climate change.

\section{Authors' contributions}

Conceived and designed the experiments: MA Saand, AA Mirbahar

Performed the experiments: SA Khaskheli \& NK Khaskheli, Analyzed the data: KA Ansari \& MM Jamro, Contributed materials/ analysis/ tools: SA Khaskheli \& AA Mirbahar, Wrote the paper: MH Sirohi \& MA Saand.

\section{References}

1. Ali SI (2008). Significance of flora with special reference to Pakistan. Pak J Bot 40(3): 967-971.

2. Qureshi R, Bhatti GR \& Shabbir G (2011). Floristic inventory of Pir Mehr Ali Shah Arid Agriculture University Research Farm at Koont and its surrounding areas. Pak J Bot 43(3):16791684.

3. Ames M \& Spooner DM (2008). DNA from herbarium specimens settles a controversy about origins of the European potato. Am J Bot 95(2): 252-257.

4. Lammertsma EI, de Boer HJ, Dekker HC, Dilcher DL, Lotter AF, \& Wagner-Cremer F (2011). Global CO2 rise leads to reduced maximum stomatal conductance in Florida vegetation. PNAS 108 (10): 4035-4040.

5. Badshah L, Hussain $F$ \& Sher $Z$ (2013). Floristic inventory, ecological characteristics and biological spectrum of rangeland, District Tank, Pakistan. Pak J Bot 45(4): 1159-1168.

6. Wariss HM, Mukhtar M, Anjum S,
Bhatti GR, Pirzada SA, \& Alam K (2013). Floristic Composition of the Plants of the Cholistan Desert, Pakistan. Am J Plant Sci 4: 58-65.

7. Gange M \& Ebadi A (2015). Flora, Life Form and Geographical Distribution of Plants in Meyantangan Mountain Refuge, East Dena Protected Area, Kohkiloye and Boyerahmad Province, Iran. J Appl Environ Biol Sci 5(9): 4552.

8. Ali A, Badshah L, Hussain F, \& Shinwari ZK (2016). Floristic composition and ecological characteristics of Plants of Chail valley, District Swat, Pakistan. Pak J Bot 48(3): 1013-1026.

9. Al-Hawshabi OS (2017). Floristic Composition, Life-forms and Chorotypes of Al-Asabah region, Ash Shamayatayn District, Taiz Governorate, Yemen. Feddes Repertorium 128: 4254.

10. Shaheen H, Qureshi R, Iram Z, Munir M, \& Ilyas M (2014). Floristic Diversity of Santh Saroola, Kotliattia, Rawalpindi. Pakistan. Pak J Bot 46(6): 1945-1954.

11. Qureshi R \& Bhatti GR (2005). Nara Desert, Pakistan: Part 1: Soils, Climate and Vegetation. Rangeland 27(5): 2731.

12. Qureshi R (2008). Preliminary floristic list of Chotiari Wetland Complex, Nawab Shah, Sindh, Pakistan. Pak J Bot 40(5): 2281-2288.

13. Qureshi R \& Bhatti GR (2010) Floristic inventory of Pai Forest, Nawab shah, Sindh, Pakistan. Pak J Bot 42(4): 2215-2224.

14. Ahmed A, Arif T, Amanullah M, Wasila H, Ahmad I, Amrani B, Abro SA, Channa SA, \& Jalbani ZH (2013). In-situ Conservation of Commiphorawightii: An Endangered Medicinal Shrub through Integrated Participatory Approach in the Nagarparkar Hills of Tharparkar, Pakistan. Uni J Agri Res 1(3): 103-109.

15. PMD (2017). Pakistan Meteorological Department. Govt of Pakistan. 
http://www.pmd.gov.pk/

16. Nasir E \& Ali SI (Eds.) (1970-1989). Flora of Pakistan (fascicles series 1190). Islamabad (Pakistan).

17. Ali SI \& Nasir YJ (Eds.) (1989-1991). Flora of Pakistan (Fascicle series). Islamabad (Pakistan).

18. Ali SI \& Qaiser M (Eds.) (1993-2008). Flora of Pakistan (Fascicle series). Islamabad (Pakistan).

19. Jafri SM (1966). The Flora of Karachi, The Book Corporation; Karachi (Pakistan).

20. Qureshi R (2004). Floristic and Ethnobotanical Study of Desert Nara Region, Sindh. Ph.D. Thesis. Department of Botany, Shah Abdul Latif University, Khairpur.

21. Raunkiaer C (1934). The life forms of plants and statistical plant geography. Oxford.

22. Chauudri II (1966). The vegetation and range Flora of Thar desert. W Pak For Deptt, Hyderabad.

23. Rajput MT, Ahmed B, Tahir SS, \& Bhatti NM (1991). A study of medicinal plants of Thar Desert. Sindh Uni Res J (Sci Sr) 23(1):15-26.

24. Ansari KA, Malik AA, \& Mahar AQ
(1993). Floristic list of district Khairpur. Ann. J Res Sci Sindh 1: 11-18.

25. Yaseen G, Ahmad M, Sultana S, Alharrasi A, Hussain J, Zafar M, Shafiq-Ur-Rehman (2015). Ethnobotany of Medicinal Plants in the Thar Desert (Sindh) of Pakistan. $J$ Ethnopharmacol 163:43-59.

26. Missouri Botanical Garden (2017) Flora of Pakistan: Pakistan plant database. Online available at http://www.tropicos.org/Project/Pakistan

27. Ansari KA, Mahar AR, Malik AR, Sirohi MH, Saand MA, Simair AA, \& Mirbahar AA (2017). Impact of grazing on plant biodiversity of desert area of district Khairpur, Sindh, Pakistan. J Anim Plant Sci 27(6): 19311940.

28. Reddy CS, Harikrishna P, Meena SL, Bhardwaj R, \& Sharma KC (2011). Composition of life form and biological spectrum along climatic gradient in Rajasthan, India. Inter $J$ Environ Sci 1(7): 1632-1639.

29. Meher-Homji VM (1964). Life forms and biological spectra as enharmonic criteria of aridity. J Indian Bot Soc 43: 424-430. 